\title{
Cognitive Abilities Predict Safety Performance: A Study Examining High-Speed Railway Dispatchers
}

\author{
Shi Lei, ${ }^{1,2}$ Zizheng Guo $\mathbb{D},{ }^{1,2}$ Xi Tan $\mathbb{D},,^{1,2}$ Xi Chen, ${ }^{3}$ Chengen Li, ${ }^{4}$ Jiaming Zou $\mathbb{D},{ }^{1,2}$ Shi Cao, ${ }^{5}$ \\ and Guo Feng $\mathbb{1}^{6}$ \\ ${ }^{1}$ School of Transportation and Logistics, Southwest Jiaotong University, Chengdu 611756, China \\ ${ }^{2}$ National United Engineering Laboratory of Integrated and Intelligent Transportation, Southwest Jiaotong University, \\ Chengdu 610031, China \\ ${ }^{3}$ Nottingham University Business School China, University of Nottingham Ningbo China, Ningbo, China \\ ${ }^{4}$ Dispatch Office of China Railway Chengdu Group Co. Ltd., Chengdu 610082, China \\ ${ }^{5}$ Department of Systems Design Engineering, University of Waterloo, Waterloo N2L 3G1, Canada \\ ${ }^{6}$ Psychological Research and Counseling Center, Southwest Jiaotong University, Chengdu 611756, China
}

Correspondence should be addressed to Guo Feng; fengguo@swjtu.edu.cn

Received 26 February 2021; Revised 4 April 2021; Accepted 19 May 2021; Published 7 June 2021

Academic Editor: Xinqiang Chen

Copyright (C) 2021 Shi Lei et al. This is an open access article distributed under the Creative Commons Attribution License, which permits unrestricted use, distribution, and reproduction in any medium, provided the original work is properly cited.

Cognitive abilities are good predictors of safety performance in many occupations. However, this correlation has not been studied from the perspective of high-speed railway (HSR) dispatchers who play a vital role in ensuring the safety and punctuality of HSR transportation system. Therefore, studying factors affecting HSR dispatchers' safety performance is not only of great importance in filling the theoretical gap, but also conducive to the selection and training of dispatchers, contributing to the reduction of human errors and the prevention of railway accidents. In this study, a total of 118 HSR dispatchers from a branch of China Railway were recruited to complete the tests that examined their cognitive abilities related to the dispatching job, including logical reasoning, visual multiobject tracking, working memory, task switching, and cognitive flexibility. Safety performance, including both the safety evaluation score obtained from the dispatchers' monthly safety performance record of the Railway Bureau and the emergency disposal performance indicated by train delay time, was evaluated with a dispatch simulator. The results suggested that better abilities in visual multiobject tracking, working memory, task switching, and cognitive flexibility were correlated with higher safety evaluation score (reflecting daily safety performance) and shorter train delay time (reflecting safety and efficiency in emergency disposal). No significant correlation was found in logical reasoning. These findings support the recommendation that cognitive abilities investigated as predictors of safety performance could be useful for the selection and training of HSR dispatchers.

\section{Introduction}

With the rapid development and popularity of high-speed railway (HSR) in China, HSR transports over a million passengers every day [1]. Due to its characteristics of high speed, high density, and large traffic volume, its safety and efficiency become the top priority of railway transportation. Although technical failures have been reduced dramatically over the past decades, human reliability becomes one of the major sources of accidents and injuries [2]. Previous studies have focused on HSR drivers [3]; however, dispatchers also play an important role in ensuring the safety and punctuality of HSR transportation especially in emergency situations [4]. Analysis has identified that errors made by dispatchers can be regarded as a significant predictive factor contributing to HSR accidents, which could lead to catastrophic consequences [5]. In 2011, on the Yong-Wen line operated by China State Railway Group, two trains had a rear-end collision, which resulted in 40 deaths and 172 injuries. The collision was partly caused by the HSR dispatcher's overlook 
of the malfunctioning signaling equipment and failure to send the correct order to the train operators [6]. Therefore, studying factors affecting HSR dispatchers' safety performance is not only of great importance for the selection and training of competent dispatchers, but also conducive to the reduction of human errors and the prevention of railway accidents.

Safety performance, regarded as an aspect of work performance, has been extensively studied in safety-critical industries such as HSR, in which safety is among the most important performance measures. Previous studies generally assessed safety performance in three ways: (1) subjective measures such as supervisor rating $[3,7]$ and self-reported rating [8, 9]; (2) data from simulator studies $[10,11]$; (3) naturalistic data from the job $[12,13]$. Among these three methods, subjective evaluation is highly operable, but it may be biased due to the different criteria of each person; the simulator method can reflect the work performance directly and objectively, but there is still some difference between the performance measurement experimental scenes and the actual work; and natural data can truly reflect the performance, but it is relatively difficult to obtain.

The analysis of HSR dispatchers' safety performance faces great challenges because of their highly complex work content and the lack of established research methods. Guo et al. [14] (2020) proposed two measures to assess the safety performance of HSR dispatchers. One is the subjective evaluation given by supervisors, and the other is the objective delay time measured by a high-fidelity dispatch simulator. As with all self-reported scales [8,9], subjective evaluation without an explicit description of performance criteria could be biased. In the present study, a dispatcher's monthly safety performance (i.e., safety evaluation score) was used to reflect daily safety performance. Monthly safety performance combines naturalistic job data (i.e., how well the dispatcher followed dispatching rules and standards required by the Railway Bureau) with supervisor rating. Thus, it has not only the high operability of subjective evaluation, but also the objective evaluation criteria that can overcome the potential deviation of subjective evaluation. In addition, we also adopted a dispatch simulator experiment to investigate HSR dispatcher safety performance (i.e., the delay time) in emergency situations to reflect dispatchers' safety performance directly and objectively.

Cognitive abilities, including both general mental ability (GMA) and specific cognitive abilities [15], are deemed to be closely correlated with high-complexity jobs [16], while they are weakly correlated with low-complexity jobs [17]. As the "central nervous system" of train operation, HSR dispatchers need to focus on scheduling, commanding, tracking, and controlling all the train operations in their jurisdiction, as well as dealing with all the emergencies quickly at any time. Hence, it is reasonable to assume that HSR dispatchers' cognitive abilities are strong predictors of their safety performance.

A growing body of literature has shown that cognitive abilities are good predictors of safety performance in many occupations such as administrative clerks, industrial workers, law enforcement officers, and military personnel
$[16,18-20]$. In the transportation industry, previous studies suggested that cognitive abilities, especially multiobject tracking and working memory, are associated with air traffic controllers' (ATC) behavior and safety performance [21, 22]. Besides, researchers have found that attention, speed anticipation, and performance stability are the significant indicators of HSR train drivers' safety performance [23].

Regarding HSR dispatchers, researchers only recently examined the relationship between dispatchers' cognitive abilities and safety performance [14]. Given the lack of relevant studies providing empirical evidence and the reality of the rapid growth of HSR, it is urgently necessary to examine the individual factors (e.g., cognitive abilities) of dispatchers' safety performance so as to form more efficient and effective selection and training systems of dispatchers $[24,25]$. Therefore, the present study aims to study the relationship between the cognitive abilities and safety performance of HSR dispatchers.

In order to identify cognitive abilities related to HSR dispatching, we analyzed Chinese HSR dispatchers' tasks based on job content analysis and related research on train dispatchers [26]. In normal conditions, dispatchers follow standard operation procedures and monitor the location and status of each train using the dispatching monitoring system. When normal operation is interrupted by bad weather, train fault, or any other emergencies, dispatchers must adjust train schedules accordingly and issue dispatching orders to implement the updated plan to address a range of potentially hazardous situations. HSR dispatchers' job responsibilities can be grouped into four categories, namely, information collection, operation monitoring, scheduling, and emergency response [14]. As shown in Figure 1, the mostly involved cognitive abilities are logical reasoning, multiobject visual tracking, working memory, task switching, and cognitive flexibility.

In the current study, we aimed to examine the correlation between cognitive abilities and HSR dispatcher safety performance. We hypothesized that cognitive abilities, including logical reasoning, multiobject visual tracking, working memory, task switching, and cognitive flexibility, are significantly correlated with HSR dispatchers' safety performance. The safety performance used both safety evaluation score that reflects daily safety performance and train delay time measured by a dispatch simulator that reflects the safety and efficiency of dispatchers' emergency disposal. The results will provide the foundation for future studies to examine the effectiveness of training methods focusing on the five specific cognitive abilities for HSR dispatchers.

\section{Materials and Methods}

2.1. Participants and Procedures. A total of 118 HSR dispatchers from a branch of China Railway participated in this study. They were all informed that participating in this academic study was voluntary, and it would not affect their performance evaluation, nor would it have any other consequences in the company. All participants were males, which is consistent with the fact that almost all HSR 


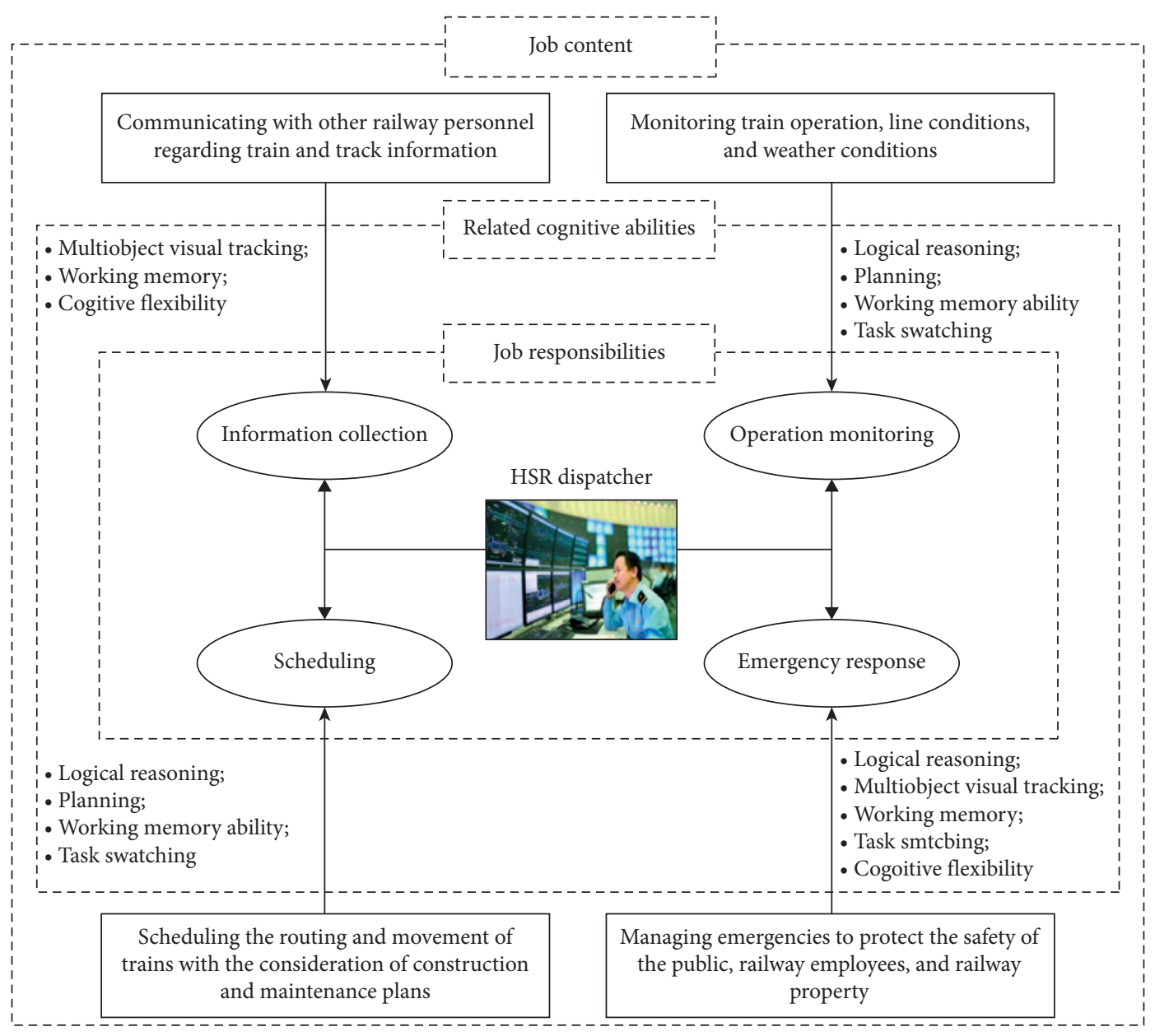

Figure 1: Chinese HSR dispatchers' job responsibilities and related cognitive abilities.

dispatchers in China are males. The participants' age ranged from 28 to 47 years (average $=36.25, \mathrm{SD}=3.96$ years). Their HSR dispatching experience ranged from 1 to 10 years (average $=6.04, \mathrm{SD}=1.96$ years). Among the participants, $83 \%$ were married, and $79 \%$ had a bachelor's degree or higher. This research was approved by the Institutional Review Board at Southwest Jiaotong University.

Participants were required to avoid alcohol and caffeine on the day of participation, as well as to refrain from eating or exercising two hours before the study. All participants completed the informed consent and then a questionnaire collecting demographic information. The cognitive ability tests were conducted on the morning of the day. Participants completed five cognitive ability tests in random order, and a ten-minute break was given between the tests. The morning session lasted for about 1.5 hours. The simulator test was conducted on the afternoon of the same day, and it lasted for about two hours. Each dispatcher participant was given 200 Yuan (RMB) and a small gift for their participation. The procedure of the experiment is shown in Figure 2.

\subsection{Cognitive Ability Tests}

2.2.1. Logical Reasoning. Logical reasoning refers to a person's capability of problem solving and rational thinking
[27]. It could be generally measured by Raven's Standard Progressive Matrices (RSPM) test [28, 29]. RSPM test is a computerized nonverbal test that consists of 60 diagrams of progressive matrices, with its reliability estimated at 0.80 by Cronbach's Alpha method [30]. Participants needed to find the rules embedded in the patterns of diagrams and apply the rules in filling in the missing piece in the matrices. The final score is within the range of 0 to 60 . The higher the score, the better the logical reasoning ability. RSPM is used to test the logical reasoning ability of HSR dispatchers, which is related to emergency disposal, plan adjustment, and other operations.

2.2.2. Visual Multiobject Tracking. Multiobject tracking (MOT) task [31] is widely used to investigate a person's capability of tracking multiple visual moving objects simultaneously [32]. The whole test consists of giving practice trials and 50 formal trials. A conceptual illustration of one trial is shown in Figure 3. Participants were required to visually track target objects (colored red in the cue-target phase and turned green in the attentive tracking phase) among nontargets (colored green), while all the objects were moving. When all the objects stopped moving, participants needed to press buttons to judge whether one randomly selected object (marked in white color) was a target or not. 


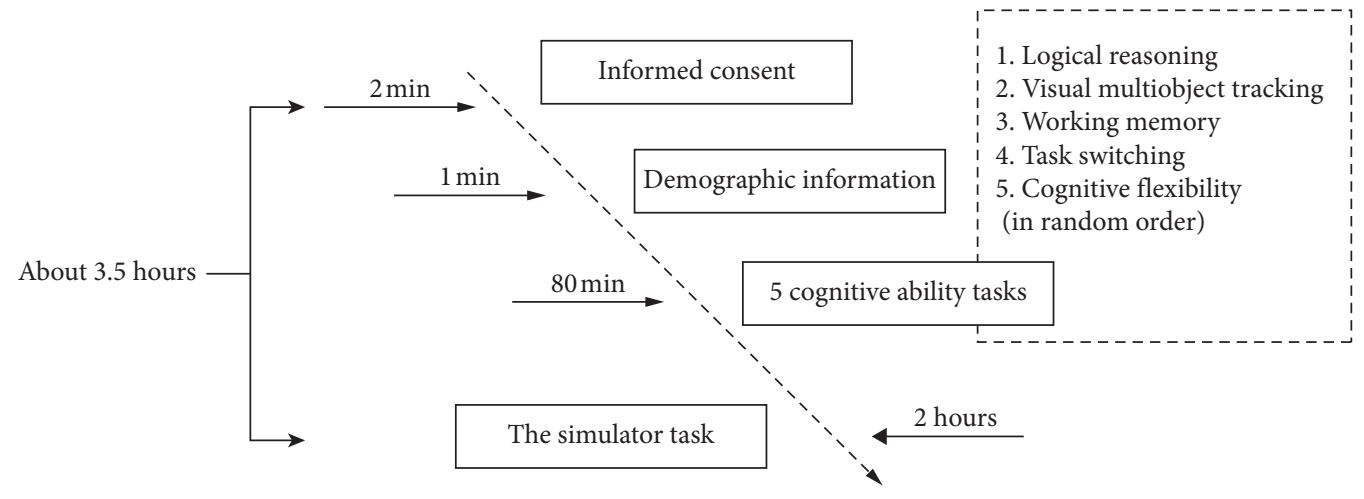

FIGURE 2: The procedure of the study.

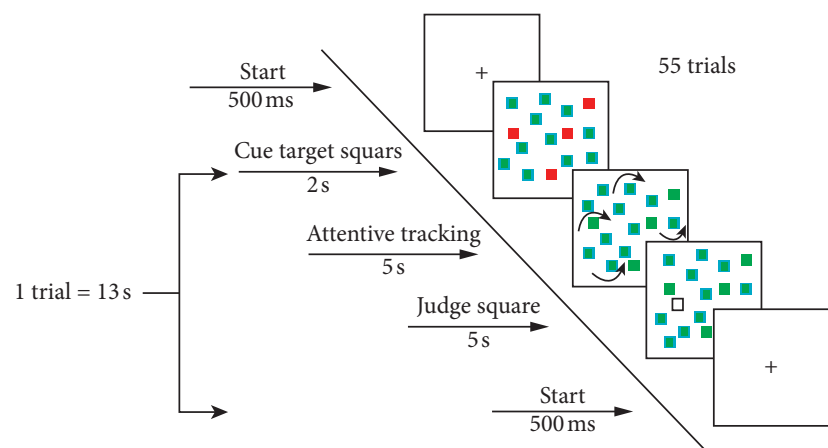

Figure 3: Conceptual illustration of MOT.

The number of targets was randomly determined ranging from one to seven, and performance was measured by both accuracy (ACC) and reaction time (RT).

2.2.3. Working Memory. The working memory, defined as a person's capacity to temporarily store and process information, is most frequently assessed by the 2-back task (e.g., [33-35]). In each trial (see Figure 4), participants were provided with a series of letters (duration was 1s) and were asked to determine whether the current letter is the same as the letter that was presented two items back in the series. If the two letters were the same, participants should give correct responses by pressing "Enter" key as quickly as possible. The whole task included 20 practice trials and 180 formal trials. The 2-back task was used to test the working memory ability of HSR dispatchers, which is related to information collection and issuing dispatching orders. Performance was measured by both accuracy (ACC) and reaction time $(\mathrm{RT})$.

2.2.4. Task Switching. The task switching test evaluates a person's ability to quickly switch from one cognitive task to another [36]. Specifically, it was used to test the HSR dispatchers' ability to switch between different job activities. As shown in Figure 5, a total of 30 practice trials and 240 formal trials were conducted. In each trial, a blue or red square was presented in the color judging phase, and one to nine numbers were presented in the number judge phase. When

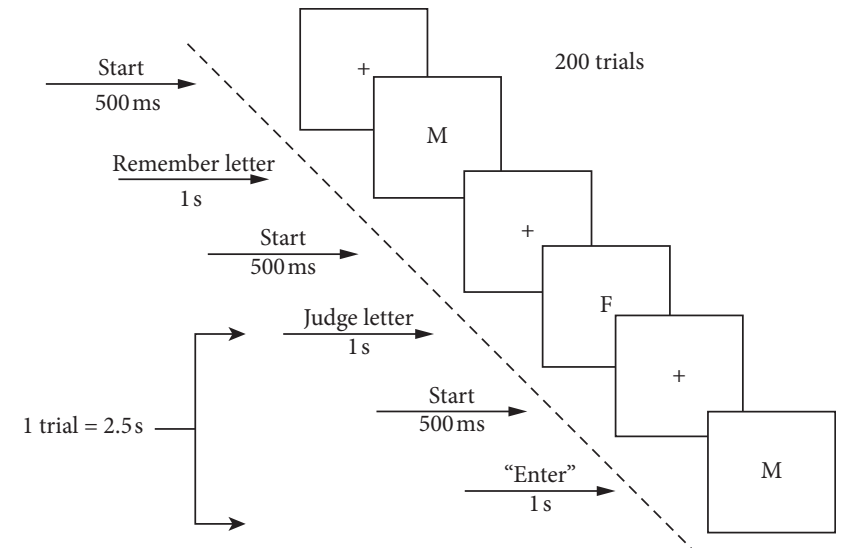

FIGURE 4: Conceptual illustration of the 2-back task.

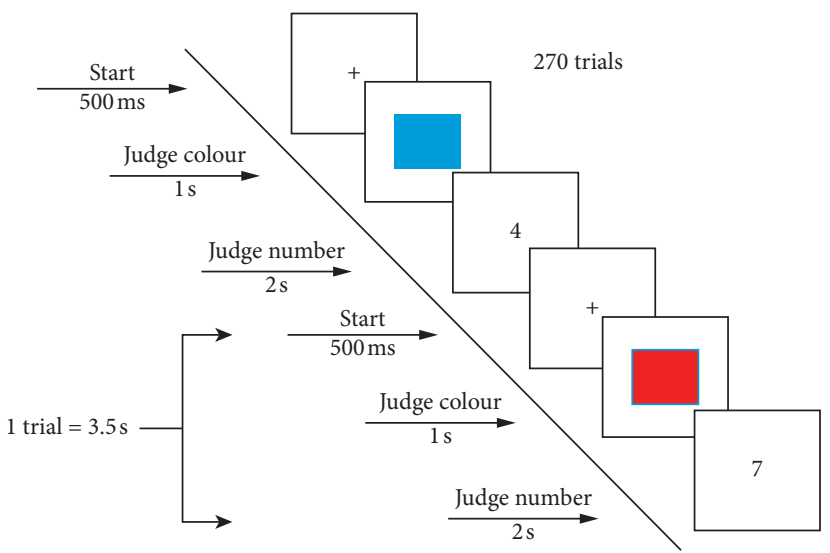

Figure 5: Conceptual illustration of the task switching test.

the blue square appears, participants were required to quickly judge the parity of the subsequent number (even or odd); when the red square appears, they were required to quickly judge whether the subsequent number is greater than or less than 5. As the participants' accuracy scores were all higher than $90 \%$, performance was measured only by reaction time of correct trials, with shorter reaction time meaning better.

2.2.5. Cognitive Flexibility. Cognitive flexibility was used to evaluate a person's capacity to construct their own 
knowledge in a variety of ways simultaneously, so that they can respond appropriately when the situation changes fundamentally [37]. In the test, as shown in Figure 6, a table with randomly positioned numbers from "1" to "11" and letters from "A" to " $K$ " was provided to participants, and they were asked to click strictly in the order "1-A-2-B-3-C." The task would not proceed if a wrong item was clicked, and participants must find the correct item and click it. Therefore, no accuracy data were collected from this task. The average reaction time from 15 formal trials was used as the measurement, with shorter reaction time meaning better.

\subsection{Safety Performance Measurements}

2.3.1. Naturalistic Data of Safety Evaluation Score. Dispatchers' safety evaluation, including information collection, operation monitoring, scheduling, and emergency response, was obtained through "HSR dispatchers' job evaluation book" of the Railway Bureau. The base score of safety evaluation was 100 for each dispatcher, and it would be increased as a result of award or decreased as a result of penalty according to the HSR dispatcher's daily safety behavior. Specific rules are shown in Table 1. The safety evaluation was conducted monthly, and the data used in the current study were the average from January to June 2020, which is as the same period of all the cognitive ability tests and simulator task conducted.

2.3.2. Train Delay Time from a Dispatch Simulator Task. Train operation would be stopped or slowed down in case of emergencies such as severe weather, equipment failure, or human failure. Such events would delay train operation for minutes or even hours. Dispatchers need to handle the emergency and ensure all the trains are running safely and on time as much as possible. Therefore, train delay time, which reflects dispatching efficiency, is an objective measure to evaluate HSR dispatchers' performance.

A high-fidelity dispatch simulator [14] was used to measure train delay time. The dispatch simulator (Figure 7) was a replicate of real dispatch control systems used in Chinese HSR. The hardware includes two PC computers (Precision 490, Dual Core Intel Xeon Processor 5130 at $2 \mathrm{GHz}$ ) and eight displays (27-inch, 1920×1200 resolution). In addition, a tablet computer that could respond automatically to the participant's speaking was used to simulate the dispatcher's communication with other railway personnel such as train drivers.

Participants (dispatchers) were asked to monitor the system and resolve problems. The emergency scenario used in the current study, which was designed by highly experienced HSR dispatchers, was an equipment failure problem when a railroad switch indicator lost its signal. Participants were instructed to resolve the problem following the emergency operating procedure and safety regulations as shown in Figure 8. The figure also shows the procedure of the dispatch simulator task. In this task, participants were asked to resume normal train operation as soon and safe as

\begin{tabular}{|c|c|c|c|c|c|c|c|}
\hline & D & & & & K & & 7 \\
\hline 3 & & & 9 & & & E & \\
\hline I & & & & A & 4 & & \\
\hline & & 5 & & & & & J \\
\hline & F & & & G & 8 & & \\
\hline & & 2 & & & & & 1 \\
\hline & B & & & 11 & & C & \\
\hline 10 & & & H & & 6 & & \\
\hline
\end{tabular}

Figure 6: Conceptual illustration of the trails test.

possible after the emergency happened. The total delay time of trains in the dispatcher's jurisdiction was calculated and used as a measure of the dispatcher's performance. Since dispatchers should put safety first, and it is extremely rare to have any accidents in this task, this delay time measurement can indicate a dispatcher's capability of response efficiency performance.

\section{Results}

3.1. Descriptive Analysis. The mean, standard deviation, minimum, and maximum values of two demographic variables, seven cognitive ability variables, and two safety performance variables are shown in Table 2.

3.2. Correlation Analysis. Two-tailed Pearson correlation was performed to examine the relationship among independent variables (including demographic variables and cognitive abilities) and dependent variables (safety evaluation score and train delay time) (Table 3). The results showed significant correlation between safety evaluation scores and train delay time $(r=-0.75, p<0.01)$, indicating that the two measurements of safety performance are of high reliability.

Furthermore, the safety evaluation score was positively correlated with the accuracy of multiobject tracking $(r=0.40, p<0.01)$ and working memory $(r=0.41, p<0.01)$; and it was negatively correlated with the reaction time of multiobject tracking $(r=-0.54, p<0.01)$, working memory $(r=-0.48, p<0.01)$, task switching $(r=-0.56, p<0.01)$, and cognitive flexibility $(r=-0.30, p<0.01)$. While delay time was positively correlated with the reaction time of multiobject visual tracking $(r=0.59, p<0.01)$, working memory $(r=0.63, p<0.01)$, task switching $(r=0.57$, $p<0.01)$, and cognitive flexibility $(r=0.35, p<0.01)$, it negatively correlated with the accuracy of multiobject visual tracking $(r=-0.42, p<0.01)$ and working memory $(r=-0.43, p<0.01)$, indicating that better cognitive abilities were associated with higher safety performance. No significant correlation was found in the relationship of logical reasoning and safety performance composed of safety evaluation score and train delay time. 
TABLE 1: The award and penalty scores within HSR dispatcher's job evaluation.

\begin{tabular}{|c|c|c|c|}
\hline Items & Events & Executive branches & Score change \\
\hline $\begin{array}{l}\text { Award items } \\
\text { (increase score) }\end{array}$ & $\begin{array}{l}\text {-- The valuable proposal was adopted } \\
\text {-- Preventing accidents or mitigating impact } \\
\text {-- Other cases }\end{array}$ & $\begin{array}{c}\text { China State Railway } \\
\text { Group Co., Ltd. } \\
\text { Railway Bureau } \\
\text { Dispatching station } \\
\quad-\end{array}$ & $\begin{array}{c}30 \\
20 \\
10 \\
1-50 \\
\end{array}$ \\
\hline $\begin{array}{l}\text { Penalty items } \\
\text { (reduce score) }\end{array}$ & $\begin{array}{l}\text {-- Class A violations occur, such as the failure } \\
\text { to send the correct order } \\
\text {-- Class B violations occur, such as the improper } \\
\text { dispatching of passenger trains } \\
\text {-- Class C violations occur, such as the improper } \\
\text { dispatching of freight trains } \\
\text {-- Class D violations occur, such as } \\
\text { incomplete dispatching order } \\
\text {-- Other cases }\end{array}$ & $\begin{array}{l}- \\
- \\
- \\
-\end{array}$ & $\begin{array}{c}-10 \\
-7.5 \\
-4 \\
-1.5 \\
-1--50\end{array}$ \\
\hline
\end{tabular}

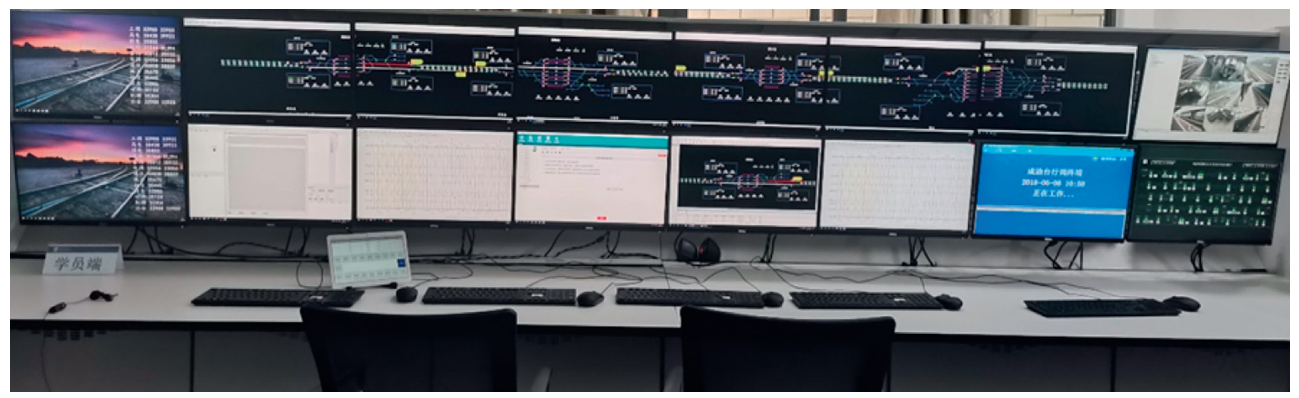

FIGURE 7: HSR dispatch simulator used to measure train delay time.

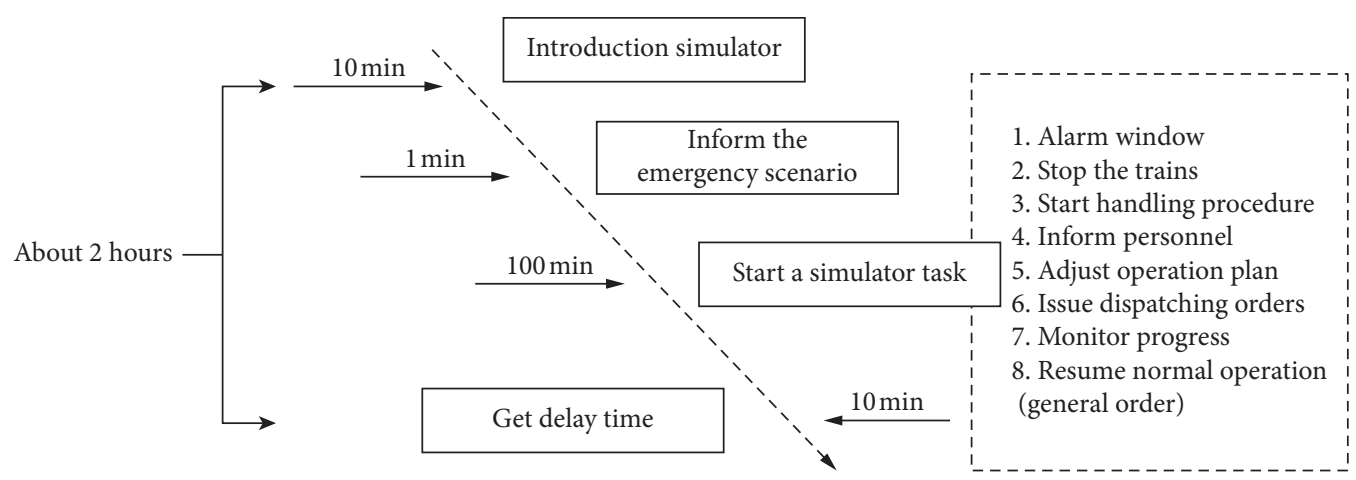

FIgURE 8: The procedure of the dispatch simulator task.

3.3. Hierarchical Regression Analysis. A two-step hierarchical regression model was established to identify the variables contributing to safety evaluation and train delay time separately. Demographic variables, including age and dispatching experience, were entered first; then cognitive abilities, including logical reasoning (score), visual multiobject tracking (both ACC and RT), working memory (both ACC and RT), task switching (RT), and cognitive flexibility (RT), were entered. As shown in Table 4, better cognitive abilities, including the accuracy of multiobject visual tracking $(\beta=0.193, p<0.05)$, working memory $(\beta=0.204$, $p<0.05)$, and the reaction time of task switching $(\beta=-0.292, p<0.01)$, could significantly predict higher safety evaluation scores, accounting for $48.4 \%$ of the total variance of the safety evaluation scores when age and dispatching experience were controlled.

Regarding the train delay time, similar results are shown in Table 5. Cognitive abilities, including accuracy of multiobject visual tracking $(\beta=-0.137, p<0.05)$, working memory $(\beta=-0.188, p<0.01)$, reaction time of working memory $(\beta=0.335, \quad p<0.01)$, task switching $(\beta=0.188$, $p<0.05)$, and cognitive flexibility $(\beta=0.137, p<0.05)$, were verified to be significant predictors of train delay time. These five independent variables could account for $59.9 \%$ of the total variance of train delay time when age and dispatching experience were controlled. 
TABLE 2: Descriptive statistics of all variables $(n=118)$.

\begin{tabular}{|c|c|c|c|c|}
\hline Variable & Mean & $\mathrm{SD}$ & Min & Max \\
\hline \multicolumn{5}{|l|}{ Control variables } \\
\hline Age & 36.25 & 3.96 & 28 & 47 \\
\hline Dispatching experience & 6.04 & 1.95 & 1 & 10 \\
\hline \multicolumn{5}{|l|}{ Cognitive ability variables } \\
\hline LR score & 37.36 & 8.46 & 21 & 58 \\
\hline ACC of MOT & 0.60 & 0.14 & 0.30 & 0.84 \\
\hline RT of MOT (ms) & 1213.68 & 296.29 & 593.69 & 1995.92 \\
\hline $\mathrm{ACC}$ of WM & 0.71 & 0.17 & 0.47 & 1.00 \\
\hline RT of WM (ms) & 696.81 & 199.14 & 408.51 & 998.52 \\
\hline $\mathrm{RT}$ of TS (ms) & 1286.21 & 297.79 & 695.69 & 2125.21 \\
\hline RT of CF (ms) & 43013.18 & 3521.36 & 37427.01 & 51235.75 \\
\hline \multicolumn{5}{|l|}{ Safety performance variables } \\
\hline SE score & 82.62 & 12.13 & 55.50 & 110.00 \\
\hline Train delay time (minute) & 227.42 & 52.55 & 123.00 & 317.00 \\
\hline
\end{tabular}

$\mathrm{LR}=$ logical reasoning; $\mathrm{MOT}=$ multiobject tracking; $\mathrm{WM}=$ working memory; $\mathrm{TS}=$ task switching; $\mathrm{CF}=$ cognitive flexible; $\mathrm{SE}=$ safety evaluation .

TABLE 3: Correlation between variables.

\begin{tabular}{|c|c|c|c|c|c|c|c|c|c|c|c|}
\hline Variable & 1 & 2 & 3 & 4 & 5 & 6 & 7 & 8 & 9 & 10 & 11 \\
\hline 1.Age & - & & & & & & & & & & \\
\hline 2. Experience & $0.53^{* *}$ & - & & & & & & & & & \\
\hline 3. LR score & 0.01 & -0.04 & - & & & & & & & & \\
\hline 4. ACC of MOT & 0.11 & 0.16 & 0.03 & - & & & & & & & \\
\hline 5. RT of MOT & 0.08 & -0.01 & -0.13 & $-0.28^{* *}$ & - & & & & & & \\
\hline 6. ACC of WM & 0.10 & 0.16 & -0.15 & $0.20^{*}$ & $-0.43^{* *}$ & - & & & & & \\
\hline 7. RT of WM & -0.01 & -0.05 & -0.15 & $-0.38^{* *}$ & $0.54^{* *}$ & $-0.20^{*}$ & - & & & & \\
\hline 8. RT of TS & -0.01 & -0.02 & -0.16 & $-0.21^{*}$ & $0.66^{* *}$ & $-0.32^{* *}$ & $0.46^{* *}$ & - & & & \\
\hline 9. RT of CF & 0.06 & 0.00 & 0.04 & $-0.20^{*}$ & $0.21^{*}$ & -0.14 & $0.25^{* *}$ & $0.28^{* *}$ & - & & \\
\hline 10. SE score & -0.02 & 0.07 & 0.11 & $0.40^{* *}$ & $-0.54^{* *}$ & $0.41^{* *}$ & $-0.48^{* *}$ & $-0.56^{* *}$ & $-0.30^{* *}$ & - & \\
\hline 11. Delay time & -0.09 & -0.14 & -0.11 & $-0.42^{* *}$ & $0.59^{* *}$ & $-0.43^{* *}$ & $0.63^{* *}$ & $0.57^{* *}$ & $0.35^{* *}$ & $-0.75^{* *}$ & - \\
\hline
\end{tabular}

${ }^{*} p<0.05 ;{ }^{* *} p<0.01$.

TABLE 4: Hierarchical regression coefficients for the prediction of safety evaluation.

\begin{tabular}{|c|c|c|c|c|c|c|}
\hline \multirow{2}{*}{ Variable } & \multicolumn{3}{|c|}{ Model 1} & \multicolumn{3}{|c|}{ Model 2} \\
\hline & $B$ & SE B & $\beta$ & $B$ & $S E B$ & $\beta$ \\
\hline Age & -0.235 & 0.336 & -0.077 & -0.199 & 0.254 & -0.065 \\
\hline Dispatching experience & 0.654 & 0.679 & 0.106 & 0.144 & 0.513 & 0.023 \\
\hline LR score & & & & 0.080 & 0.104 & 0.056 \\
\hline RT of MOT & & & & -0.004 & 0.004 & -0.087 \\
\hline ACC of MOT & & & & 16.579 & 6.592 & $0.193^{*}$ \\
\hline RT of WM & & & & -0.010 & 0.005 & -0.158 \\
\hline ACC of WM & & & & 14.677 & 5.793 & $0.204^{*}$ \\
\hline RT of TS & & & & -0.012 & 0.004 & $-0.292^{* *}$ \\
\hline RT of CF & & & & 0.000 & 0.000 & -0.092 \\
\hline $\mathrm{R}^{2}$ & & 0.008 & & & 0.484 & \\
\hline F For change in $R^{2}$ & & 0.489 & & & $11.275^{* *}$ & \\
\hline
\end{tabular}

$\mathrm{LR}=$ logical reasoning; $\mathrm{MOT}=$ multiobject tracking; $\mathrm{WM}=$ working memory; $\mathrm{TS}=$ task switching; $\mathrm{CF}=$ cognitive flexibility; $\mathrm{SE}=$ safety evaluation. ${ }^{*} p<0.05$; ${ }^{* *} p<0.01$.

\section{Discussion}

The aim of this study was to explore the influence of cognitive abilities on HSR dispatchers' safety performance. By measuring the five cognitive abilities and the two safety performance indicators, we tested 118 HSR dispatchers. The current results revealed that the related cognitive abilities were correlated with participants' safety performance.
Cognitive abilities composed of visual multiobject tracking, working memory, task switching, and cognitive flexibility could positively predict the safety performance indicators, namely, safety evaluation score and train delay time, which explained $48.4 \%$ and $59.9 \%$ of the total variance in the hierarchical regression, respectively, indicating that the stronger abilities of visual multiobject tracking, working memory, task switching, and cognitive flexibility were 
TABLE 5: Hierarchical regression coefficients for the prediction of train delay time.

\begin{tabular}{|c|c|c|c|c|c|c|}
\hline \multirow{2}{*}{ Variable } & \multicolumn{3}{|c|}{ Model 1} & \multicolumn{3}{|c|}{ Model 2} \\
\hline & $B$ & $S E B$ & $\beta$ & $B$ & SE B & $\beta$ \\
\hline Age & -0.164 & 1.445 & -0.012 & -0.547 & 0.970 & -0.041 \\
\hline Dispatching experience & -3.696 & 2.923 & -0.138 & -1.359 & 1.958 & -0.051 \\
\hline LR score & & & & -0.252 & 0.398 & -0.041 \\
\hline RT of MOT & & & & 0.024 & 0.017 & 0.138 \\
\hline ACC of MOT & & & & -50.814 & 25.177 & $-0.137^{*}$ \\
\hline RT of WM & & & & 0.088 & 0.020 & $0.335^{* *}$ \\
\hline ACC of WM & & & & -58.591 & 22.123 & $-0.188^{* *}$ \\
\hline RT of TS & & & & 0.033 & 0.015 & $0.188^{*}$ \\
\hline RT of CF & & & & 0.002 & 0.001 & $0.137^{*}$ \\
\hline $\mathrm{R}^{2}$ & & 0.021 & & & 0.599 & \\
\hline F For change in $R^{2}$ & & 1.230 & & & $17.927^{* *}$ & \\
\hline
\end{tabular}

$\mathrm{LR}=$ logical reasoning; $\mathrm{MOT}=$ multiobject tracking; $\mathrm{WM}=$ working memory; $\mathrm{TS}=$ task switching; $\mathrm{CF}=$ cognitive flexibility; $\mathrm{SE}=$ safety evaluation. ${ }^{*} p<0.05$; ${ }^{* *} p<0.01$.

associated with better safety performance of HSR dispatchers, while the correlations between logical reasoning and both safety performance were not significant. These findings can provide evidence and indicators for the selection and training of HSR dispatchers.

Consistent with existing studies [38], the positive correlation between visual multiobject tracking and dispatchers' safety performance is verified in the present study. Based on the literature $[26,39]$ and task analysis, HSR dispatchers need to monitor the operational status of multiple trains in their jurisdiction, as well as the conditions of signals, stations, switches, and weather. Dynamic visual tracking of multiple objects becomes an indispensable part of their daily work [38]. Therefore, it is understandable that visual MOT ability is an effective predictor of HSR dispatchers' performance.

The working memory ability often correlates with task performance such as school grades and group performance $[40,41]$. Working memory is an important requirement in HSR dispatchers' job because they need to process multiple pieces of information and synthesize them in decision making. The current results confirmed that it was a good predictor of dispatchers' safety performance. Hence, the Railway Bureau should pay more attention to dispatchers' working memory ability in the selection and training processes.

Consistent with the results from other jobs that also require multitasking $[24,42,43]$, the findings from the current study confirmed that task switching ability and cognitive flexibility are important predictors of safety performance (train delay time). Task switching ability is the capacity to switch between different cognitive tasks [36], and cognitive flexibility is a key aspect of mental executive process, namely, the deployment of cognitive resources to adapt to changes in events [44]. HSR dispatchers serve as a central commander who needs to organize other railway personnel and resources. They frequently switch between sending dispatching commands, making phone calls, monitoring train operation, and other tasks for almost the entirety of their working hours. Therefore, their task switching and cognitive flexibility have considerable impact on their dispatching accuracy and efficiency [45]. All these results recommend that cognitive abilities should be considered in the prediction of safety performance [46].

Inconsistent with the work analysis and the study hypothesis, the ability of logical reasoning does not show significant correlation with safety performance indicators (safety evaluation score and train delay time). A potential explanation is that dispatchers' performance has a low dependency on their educative and productive aspects of mental ability measured by RSPM [27-29]. Dispatchers' job is highly proceduralized and standardized. Although HSR dispatching is a task with multiple pieces of information and current task requirements, especially in emergency situations, it has clear procedures provided in manuals and regulations to guide problem solving, and that may reduce the association [17].

There are still some limitations in the current study. Firstly, all the HSR dispatchers were chosen just from only two railway group. Future studies should examine the predictive model among more widen dispatcher populations such as groups in other geographical regions or other countries. Secondly, as for logical reasoning, more targeted method should be adopted for further investigating. Lastly, in addition to the five cognitive indicators, future research should conduct a more in-depth analysis of dispatchers' work content to search for other cognitive factors that may also affect their safety performance. And other factors such as work attitude, personality, and adventure motivation should also be taken into account in subsequent studies.

\section{Conclusions}

In summary, the results from the current study support that the cognitive abilities, including multiobject visual tracking, working memory, task switching, and cognitive flexibility abilities, are positive predictors of safety performance (both subjective and objective measures) among Chinese HSR dispatchers. These findings support the recommendation that the cognitive abilities investigated as predictors could be useful for the selection and training of HSR dispatchers. Besides, safety performance (safety evaluation score and train delay time) has a high degree of consistency, which could also serve as a tool for the selection of dispatchers. 
Beside cognitive abilities, other factors such as personality and motivation may also be important predictors of safety performance. Future studies are needed to further examine additional factors.

\section{Data Availability}

All data and models used during the study are available from the corresponding author by request.

\section{Conflicts of Interest}

The authors declare that there are no conflicts of interest regarding the publication of this paper.

\section{Authors' Contributions}

Shi Lei and Zizheng Guo contributed equally to this manuscript.

\section{Acknowledgments}

This study was supported by the National Natural Science Foundation of China (32000734, 51108390, 71601163), Science and Technology Program of China Railway (2018F024), Science and Technology Program of Sichuan Province (2019YFG0043), and Science and Technology Innovation Project of Shuohuang Railway Development Co., Ltd. (SHTL-19-01).

\section{References}

[1] X. Dong, "High-speed railway and urban sectoral employment in China," Transportation Research Part A: Policy and Practice, vol. 116, pp. 603-621, 2018.

[2] K. Pazouki, N. Forbes, R. A. Norman, and M. D. Woodward, "Investigation on the impact of human-automation interaction in maritime operations," Ocean Engineering, vol. 153, pp. 297-304, 2018.

[3] W. Wei, M. Guo, L. Ye, G. Liao, and Z. Yang, "Work-family conflict and safety participation of high-speed railway drivers: job satisfaction as a mediator," Accident Analysis \& Prevention, vol. 95, pp. 97-103, 2016.

[4] W. Wang, X. Liu, and Y. Qin, "A modified HEART method with FANP for human error assessment in high-speed railway dispatching tasks," International Journal of Industrial Ergonomics, vol. 67, pp. 242-258, 2018.

[5] Y. Sun, Q. Zhang, Z. Yuan, Y. Gao, and S. Ding, "Quantitative analysis of human error probability in high-speed railway dispatching tasks," IEEE Access, vol. 8, pp. 56253-56266, 2020.

[6] Y. Fan, Z. Li, J. Pei, H. Li, and J. Sun, "---Applying systems thinking approach to accident analysis in China: case study of "7.23" Yong-Tai-Wen High-Speed train accident," Safety Science, vol. 76, pp. 190-201, 2015.

[7] J. Zhang, Y. Li, and C. Wu, "The influence of individual and team cognitive ability on operators' task and safety performance: a multilevel field study in nuclear power plants," PLOS ONE, vol. 8, no. 12, Article ID e84528, 2014.

[8] C.-E. Havârneanu, C. Măirean, and S.-A. Popuşoi, "Workplace stress as predictor of risky driving behavior among taxi drivers. The role of job-related affective state and taxi driving experience," Safety Science, vol. 111, pp. 264-270, 2019.
[9] V. Linkov, A. Zaoral, P. Řezáč, and C.-W. Pai, "Personality and professional drivers' driving behavior," Transportation Research Part F: Traffic Psychology and Behaviour, vol. 60, pp. 105-110, 2019.

[10] T. W. Casey and A. D. Krauss, "The role of effective error management practices in increasing miners' safety performance," Safety Science, vol. 60, pp. 131-141, 2013.

[11] D. Pan, Y. Zhang, and Z. Li, "Predictive capability of cognitive ability and cognitive style for spaceflight emergency operation performance," International Journal of Industrial Ergonomics, vol. 54, pp. 48-56, 2016.

[12] X. Chen, L. Qi, Y. Yang et al., "Video-based detection infrastructure enhancement for automated ship recognition and behavior analysis," Journal of Advanced Transportation, vol. 2020, Article ID 7194342, 12 pages, 2020.

[13] M. Guo, W. Wei, G. Liao, and F. Chu, "The impact of personality on driving safety among Chinese high-speed railway drivers," Accident Analysis \& Prevention, vol. 92, pp. 9-14, 2016.

[14] Z. Guo, J. Zou, C. He, X. Tan, C. Chen, and G. Feng, “The importance of cognitive and mental factors on prediction of job performance in Chinese high-speed railway dispatchers," Journal of Advanced Transportation, vol. 2020, Article ID 7153972, 13 pages, 2020.

[15] N. Blacksmith, T. S. Behrend, R. S. Dalal, and T. L. Hayes, "General mental ability and decision-making competence: theoretically distinct but empirically redundant," Personality and Individual Differences, vol. 138, pp. 305-311, 2019, https:// doi.org/10.1016/j.paid.2018.10.024.

[16] J. F. Salgado, N. Anderson, S. Moscoso, C. Bertua, F. de Fruyt, and J. P. Rolland, "A meta-analytic study of general mental ability validity for different occupations in the European community," Journal of Applied Psychology, vol. 88, no. 6, pp. 1068-1081, 2003.

[17] J. W. B. Lang, M. Kersting, U. R. Hülsheger, and J. Lang, "General mental ability, narrower cognitive abilities, and job performance: the perspective OF the nested-factors model OF cognitive abilities," Personnel Psychology, vol. 63, no. 3, pp. 595-640, 2010.

[18] C. Bertua, N. Anderson, and J. F. Salgado, "The predictive validity of cognitive ability tests: a UK meta-analysis," Journal of Occupational and Organizational Psychology, vol. 78, no. 3, pp. 387-409, 2005.

[19] F. Bosco, D. G. Allen, and K. Singh, "Executive attention: an alternative perspective on general mental ability, performance, and subgroup differences," Personnel Psychology, vol. 68, no. 4, pp. 859-898, 2015.

[20] F. Schmidt, "The role of general cognitive ability and job performance: why there cannot Be a debate," Human Performance, vol. 15, no. 1, pp. 187-210, 2002.

[21] A. R. Dattel and R. E. King, "The impact of reweighting the AT-SAT subtests on group score differences," The International Journal of Aviation Psychology, vol. 20, no. 2, pp. 111-123, 2010.

[22] A. Mojzisch, S. Krumm, and T. Schultze, "Do high working memory groups perform better?" Journal of Personnel Psychology, vol. 13, 2014.

[23] M. Guo, L. Hu, and L. Ye, "-Cognition and driving safety: how does the high-speed railway drivers' cognitive ability affect safety performance?" Transportation Research Part F: Traffic Psychology and Behaviour, vol. 65, pp. 10-22, 2019.

[24] D. Gardner and D. L. Deadrick, "Underprediction of performance for US minorities using cognitive ability measures," Equal Opportunities International, vol. 27, 2008. 
[25] A. R. Kolz, L. A. McFarland, and S. B. Silverman, "Cognitive ability and job experience as predictors of work performance," The Journal of Psychology, vol. 132, no. 5, pp. 539-548, 1998.

[26] Gertler, J. Selection of Railroad Dispatcher Candidates; 2003.

[27] J. Raven, “The raven's progressive matrices: change and stability over culture and time," Cognitive Psychology, vol. 41, no. 1, pp. 1-48, 2000.

[28] Gangat, N. Relating Academic Performance to L1 and L2 Learners' Scores on the SDRT and Raven's SPM. 2017.

[29] J. Osborne, S. Simon, A. Christodoulou, C. Howell-Richardson, and K. Richardson, "Learning to argue: a study of four schools and their attempt to develop the use of argumentation as a common instructional practice and its impact on students," Journal of Research in Science Teaching, vol. 50, no. 3, pp. 315-347, 2013.

[30] R. A. Peterson, "A meta-analysis of Cronbach's coefficient alpha," Journal of Consumer Research, vol. 21, no. 2, pp. 381-391, 1994.

[31] K. Smith, D. Gatica-Perez, J. Odobez, and B. Sileye, "Evaluating multi-object tracking," in Proceedings of the 2005 IEEE Computer Society Conference on Computer Vision and Pattern Recognition (CVPR'05) - Workshops, p. 36, San Diego, CA, USA, September 2005.

[32] G. A. Alvarez, T. S. Horowitz, H. C. Arsenio, J. S. DiMase, and J. M. Wolfe, "Do multielement visual tracking and visual search draw continuously on the same visual attention resources?" Journal of Experimental Psychology: Human Perception and Performance, vol. 31, no. 4, pp. 643-667, 2005.

[33] S. Cao, U. Reiter, and M. Weitzel, "Influence of interaction on perceived quality in audio visual applications: subjective assessment with n-back working memory task," in Proceedings of Audio Engineering Society Conference: 30th International Conference: Intelligent Audio Environments, Saariselkä, Finland, March 2007.

[34] A. M. Owen, K. M. McMillan, A. R. Laird, and E. Bullmore, "N-back working memory paradigm: a meta-analysis of normative functional neuroimaging studies," Human Brain Mapping, vol. 25, no. 1, pp. 46-59, 2005.

[35] U. Reiter and M. Weitzel, "Influence of interaction on perceived quality in audio visual applications: subjective assessment with n-back working memory task, ii," Proceedings of Audio Engineering Society Convention, vol. 122, 2020.

[36] S. Monsell, "Task switching," Trends in Cognitive Sciences, vol. 7, no. 3, pp. 134-140, 2003.

[37] A. Moore, "Mindfulness and cognitive flexibility," Consciousness and Cognition, vol. 18, pp. 176-186, 2009.

[38] A. Tazoniero, R. Gonçalves, and F. Gomide, "Decision making strategies for real-time train dispatch and control," in Analysis and Design of Intelligent Systems Using Soft Computing Techniques, P. Melin, O. Castillo, E. G. Ramírez, J. Kacprzyk, and W. Pedrycz, Eds., Springer, Berlin, Heidelberg, 2007.

[39] Devoe, D. B. An Analysis of the Job of Railroad Train Dispatcher. 1974.

[40] K. Barker, R. Allen, and P. McGeorge, "Multiple-object tracking: enhanced visuospatial representations as a result of experience," Experimental Psychology, vol. 57, pp. 208-214, 2010.

[41] W. Jiang, T. Feng, and J. Pan, "Features of multiple-objects tracking task processing in student pilots," Space Medicine \& Medical Engineering, vol. 26, pp. 269-273, 2013.

[42] P. Cheng, G. Tallent, T. J. Bender, K. M. Tran, and C. L. Drake, "Shift work and cognitive flexibility: decomposing task performance," Journal of Biological Rhythms, vol. 32, pp. 143-153, 2017.

[43] R. M. Hope, E. M. Rantanen, and L. Oksama, "Multiple identity tracking and entropy in an ATC-like task," Proceedings of the Human Factors and Ergonomics Society Annual Meeting, vol. 54, pp. 1012-1016, 2010.

[44] P. Whitney, J. M. Hinson, M. L. Jackson, and H. P. Van Dongen, "Feedback blunting: total sleep deprivation impairs decision making that requires updating based on feedback," Sleep, vol. 38, pp. 745-754, 2015.

[45] E. M. Roth, N. Malsch, J. Multer, and M. Coplen, "Understanding how train dispatchers manage and control trains: a cognitive task analysis of a distributed team planning task," Proceedings of the Human Factors and Ergonomics Society Annual Meeting, vol. 43, pp. 218-222, 1999.

[46] T. A. Judge and J. D. Kammeyer-Mueller, "General and specific measures in organizational behavior research: considerations, examples, and recommendations for researchers," Journal of Organizational Behavior, vol. 33, pp. 161-174, 2012. 